Article

\title{
Identification and Analysis of Regulatory Elements in Porcine Bone Morphogenetic Protein 15 Gene Promoter
}

\author{
Qianhui Wan ${ }^{\dagger}$, Yaxian Wang ${ }^{\dagger}$ and Huayan Wang * \\ Department of Animal Biotechnology, College of Veterinary Medicine, Northwest A\&F University, \\ Xi' an 712100, Shaanxi, China; E-Mails: wanqianhui_casey@163.com (Q.W.); \\ wangyaxianwyx@163.com (Y.W.)
}

$\dagger$ These authors contributed equally to this work.

* Author to whom correspondence should be addressed; E-Mail: hhwang101@nwsuaf.edu.cn; Tel.: +86-29-8708-0069; Fax: +86-29-8708-0068.

Academic Editor: Xiaofeng Jia

Received: 6 August 2015 / Accepted: 20 October 2015 / Published: 27 October 2015

\begin{abstract}
Bone morphogenetic protein 15 (BMP15) is secreted by the mammalian oocytes and is indispensable for ovarian follicular development, ovulation, and fertility. To determine the regulation mechanism of $B M P 15$ gene, the regulatory sequence of porcine $B M P 15$ was investigated in this study. The cloned BMP15 promoter retains the cell-type specificity, and is activated in cells derived from ovarian tissue. The luciferase assays in combination with a series of deletion of $B M P 15$ promoter sequence show that the -427 to -376 bp region of $B M P 15$ promoter is the primary regulatory element, in which there are a number of transcription factor binding sites, including LIM homeobox 8 (LHX8), newborn ovary homeobox gene (NOBOX), and paired-like homeodomain transcription factor 1 (PITX1). Determination of tissue-specific expression reveals that LHX8, but not PITX1 and NOBOX, is exclusively expressed in pig ovary tissue and is translocated into the cell nuclei. Overexpression of LHX8 in Chinese hamster ovary (CHO) cells could significantly promote $B M P 15$ promoter activation. This study confirms a key regulatory element that is located in the proximal region of BMP15 promoter and is regulated by the LHX8 factor.
\end{abstract}

Keywords: pig; BMP15; promoter; transcription factor; LHX8 


\section{Introduction}

Reproductive capability is a vital economic factor for sows, for instance the Chinese Taihu breed farrows at least five more piglets per litter than the Large White breed [1]. Many studies have revealed that oocytes can produce and secrete growth factors that facilitate follicular development [2,3]. Two growth factors, bone morphogenetic protein 15 (BMP15) and growth and differentiation factor 9 (GDF9), belonging to the transforming growth factor $\beta$ (TGF- $\beta$ ) superfamily, are produced by oocytes [4,5]. These two factors participate in the different stages of follicular development and influence the final events of embryo maturation and ovulation [6,7]. In mouse, Bmp15 homozygous mutant causes subfertile, showing the ovulation defects and reduction of oocytes [8]. In contrast to Bmp15 null mice, in sheep, the naturally-occurring homozygous mutants $\left(F e c X^{I}\right)$ that has point mutations in the coding region of BMP15 gene causes the infertile due to an arrest at the primary stage of folliculogenesis. On the other hand, the heterozygous females show increased ovulation rate and multiple pregnancies. Thus, BMP15 is related to infertility and super-fertility in a dosage-sensitive manner in sheep [9,10]. Recent reports showed that the BMP15 mRNA level in individual denuded oocytes from single-to-triple ovulation-rate species (i.e., sheep) was much lower than that from high ovulation-rate species (i.e., pig). But the ratios of BMP15:GDF9 expression levels were significantly species-specific indicating a unique mechanism by which the species-specific ovulation-rate may be regulated [11]. A tissue screening study showed that pig GDF9 is expressed not only in ovarian tissue, but also in other somatic tissues, whereas pig BMP15 is only expressed in ovary [12]. Thus, it is worth further investigation of the transcription regulation of pig BMP15 gene.

As a member of the BMP superfamily, BMP15, also called growth and differentiation factor 9B (GDF9B), plays a vital role in ovarian follicular development, ovulation and fertility [13,14]. The initiation of BMP15 gene expression occurs during early follicular development in either primordial or primary follicles depending on animal species [15]. After a series of post-translational modifications, BMP15 is secreted from oocytes and forms either homodimers (BMP15:BMP15) or heterodimers (BMP15:GDF9). Both dimers can then bind to serine/threonine kinase type I-II receptors on the surface of granulosa cells, which, in turn, activates the intracellular SMAD signaling pathway [7]. Functionally, BMP15 expression in the oocyte stimulates granulosa cell proliferation and inhibits the action of follicle-stimulating hormone (FSH) by suppressing the expression of FSH receptor, which is related to ovulation rate and fertility [16]. Following identification of the functions of BMP15 in the ovary, the progress has been made towards a molecular understanding of how this gene is regulated by other factors in oocytes. Several transcription factors such as paired-like homeodomain transcription factor 1 (PITX1), germ cell nuclear factor (GCNF), LIM homeobox 8 (LHX8) and transcriptional protein Yin Yang 1 (YY1) can regulate mouse and human BMP15 expression [17-20], however, the regulation network of pig $B M P 15$ gene has not been fully investigated yet.

To understand the regulatory mechanism of pig BMP15 gene expression, we analyzed porcine $B M P 15$ gene $5^{\prime}$ untranslated and promoter regions, screened the primary regulatory sequence, and verified the potential transcription factors that could upregulate the expression of porcine BMP15. 


\section{Results}

\subsection{Molecular Cloning and Bioinformatics Analysis of Bone Morphogenetic Protein 15 (BMP15) Promoter}

Bioinformatics analysis showed that several potential binding sites for the reproduction related factors, including germ cell nuclear factor (GCNF), LIM homeobox 8 (LHX8), newborn ovary homeobox gene (NOBOX), and paired-like homeodomain transcription factor 1 (PITX1), were found in the promoter sequence of BMP15 gene, indicating that BMP15 may be a downstream target of those transcription factors (Figure 1). We also found that there was no typical TATA box element in the proximal region of porcine BMP15 promoter. Indeed, a typical core promoter element such as the TATA box does not always exist in a basic promoter region [21]. To further investigate the expression specificity of $B M P 15$, a $2.2 \mathrm{~kb}$ promoter fragment (Accession No: KF114861.1) was subcloned into pEGFP-1 and pGL3-basic plasmid to make the reporter vector pE2.2 and pL2.2 (Figure 2A,B). The transient transfection of pL2.2 and GFP fluorescence detection showed that the BMP15 promoter was only activated in Chinese hamster ovary (CHO) cells, but not in $\mathrm{C} 2 \mathrm{C} 12$ and NIH3T3 cells (Figure 2C), indicating that the cloned $B M P 15$ promoter retained the cell-type specificity. The luciferase assays reveal the time-dependent activation of the $B M P 15$ promoter (Figure 2D).

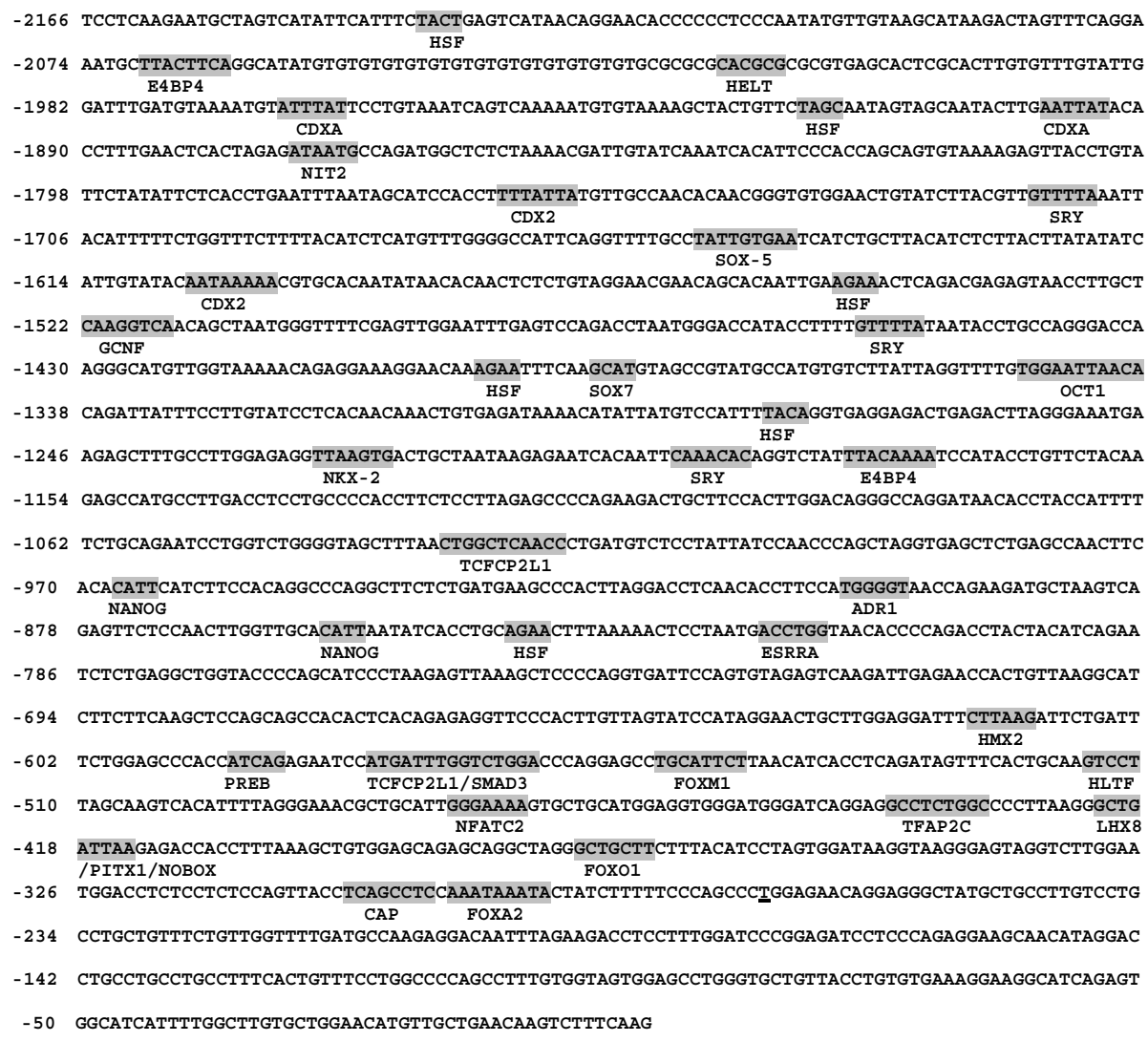

Figure 1. Porcine $B M P 15$ gene $5^{\prime}$ untranslated region (UTR) and transcription regulatory region. The $2166 \mathrm{bp} \mathrm{5'}$ UTR and promoter region of porcine BMP15 gene was cloned from porcine ovary tissue. The potential transcription initiation site is underlined. The predicted DNA binding sites are highlighted. 
A

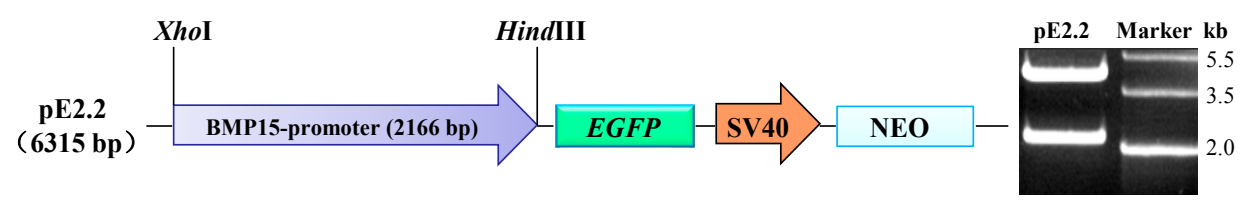

B

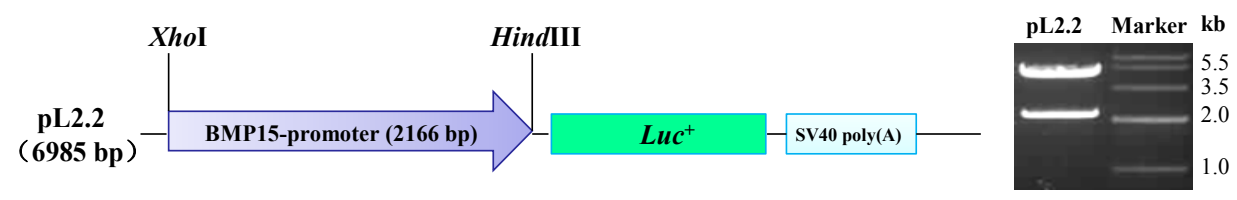

C
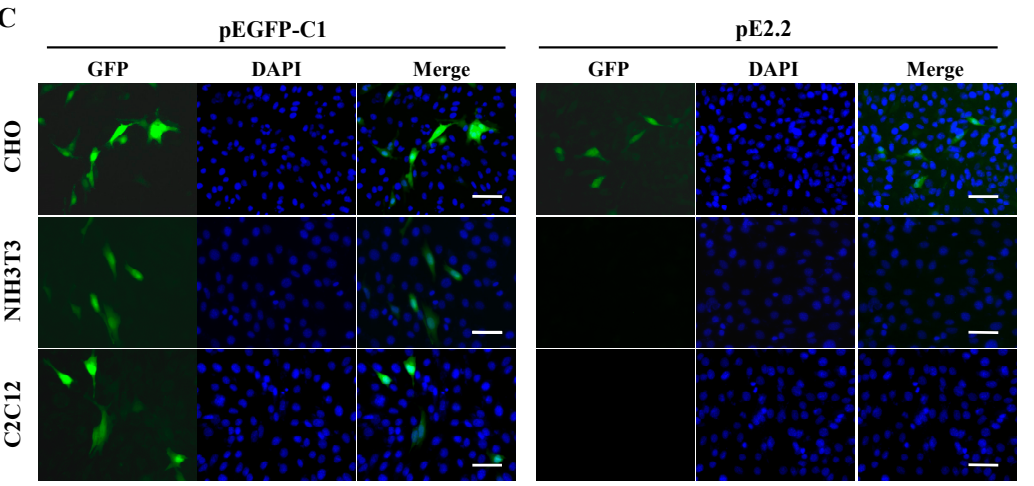

D

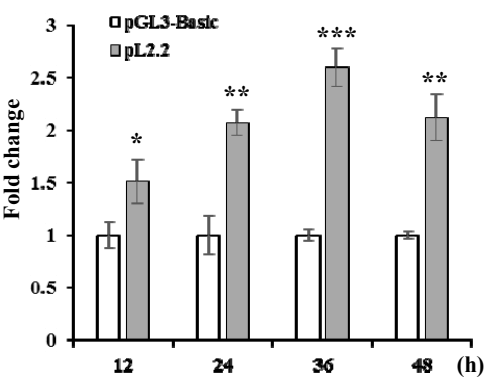

Figure 2. Functional analysis of cloned porcine $B M P 15$ promoter. (A) Reporter vector pE2.2 is derived from pEGFP-1 vector and contains $2.2 \mathrm{~kb} B M P 15$ promoter fragment that is confirmed by $\mathrm{Xho} /$ /Hind $I I I$ digestions; (B) Reporter vector pL2.2 is derived from pGL3-basic vector and contains $2.2 \mathrm{~kb}$ fragment confirmed by XhoI/Hind $I I I$ digestions; (C) Cell-specific activation of BMP15 promoter in different cell lines. Scale bar, $100 \mu \mathrm{m}$; and (D) Time-dependent luciferase assay for BMP15 promoter activity in $\mathrm{CHO}$ cells. The pGL3-Basic vector was used as control. Values are represented as mean $\pm \mathrm{SD}, * p<0.05$, $* * p<0.01, * * * p<0.001, n=3$.

\subsection{Investigation of Primary Regulatory Elements in BMP15 Promoter}

The procedures of promoter deletion and protein-DNA binding assays have been used to identify the essential promoter sequences [22,23]. In this study, we used the similar strategy to investigate the primary regulatory sequence of $B M P 15$ promoter (Figure $3 \mathrm{~A}$ ). The truncated $B M P 15$ promoter fragments $(-1886 \sim+4,-1320 \sim+4,-870 \sim+4,-589 \sim+4,-460 \sim+4,-357 \sim+4$, and $-200 \sim+4)$ were amplified from $\mathrm{pL} 2.2$, and were then inserted into pGL3-basic plasmid to construct reporter vectors pL1.8, pL1.3, pL0.8, pL0.5, pL0.4, pL0.3 and pL0.2 (Table S1). These recombinant plasmids were confirmed by $\mathrm{Xho} I$ and Hind $I I I$ double digestions (Figure 3B). The truncated constructs were transiently transfected into $\mathrm{C} 2 \mathrm{C} 12, \mathrm{NIH} 3 \mathrm{~T} 3$ and $\mathrm{CHO}$ cells, and the activity of these different BMP15 promoter constructs was determined by a dual-luciferase assay. Results showed that BMP15 only expressed in $\mathrm{CHO}$ cells, but not in $\mathrm{C} 2 \mathrm{C} 12$ or NIH3T3. The promoter activity in pL0.4 was significantly increased comparing to $\mathrm{pL} 0.3$ and $\mathrm{pL} 0.5$, suggesting that within the region of -357 to $-460 \mathrm{bp}$ existed an important positive regulatory element. In addition, we noticed that the BMP15 promoter activity was significantly reduced in pL0.5 versus $\mathrm{pL} 0.4$, and expected that this region contains a negative regulatory element that is worth investigating in the future (Figure $3 \mathrm{C}$ ). 


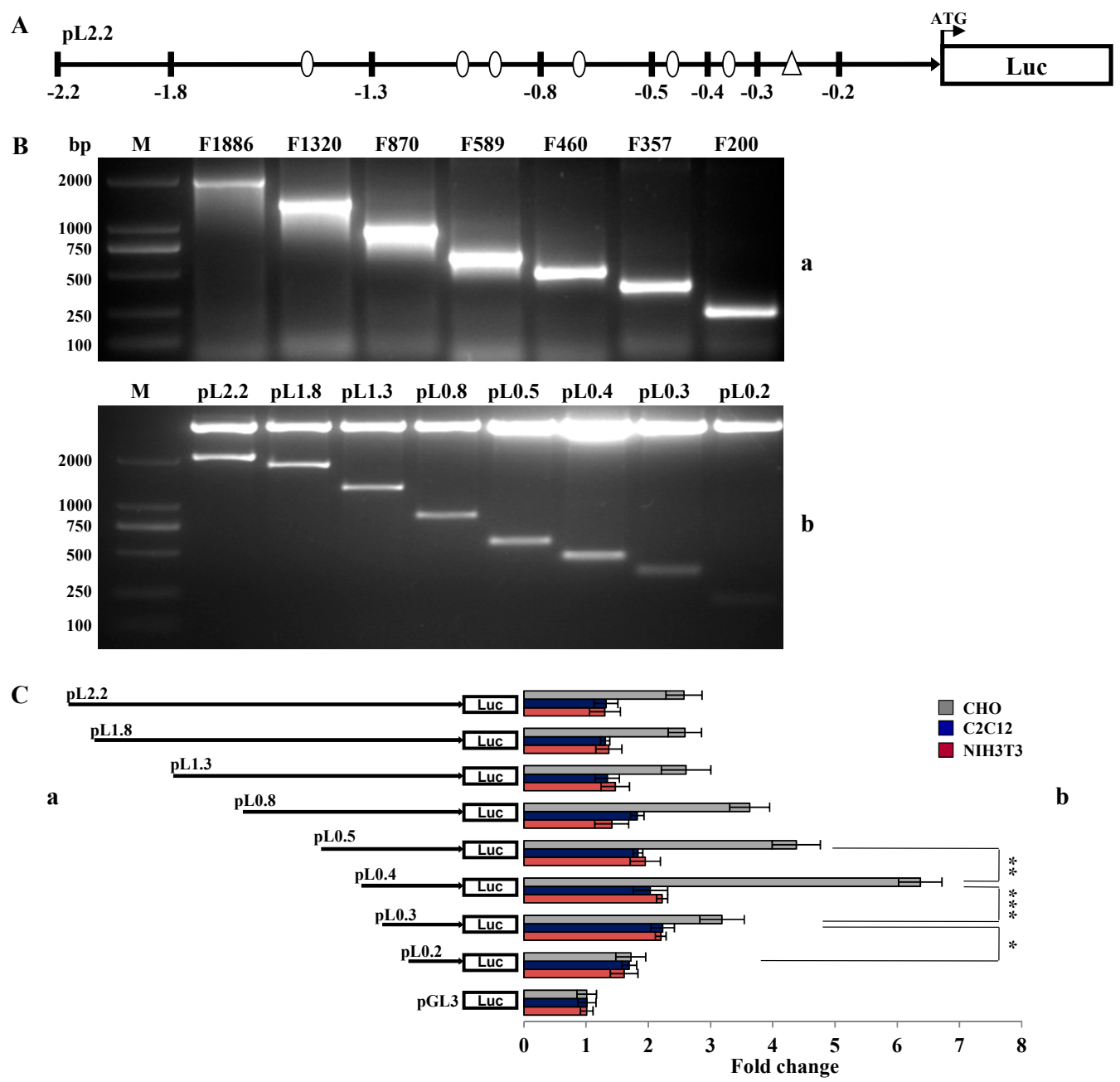

Figure 3. Screening of the core regulatory sequence in BMP15 promoter. (A) The schematic diagram of vector pL2.2. The sites of truncated constructs are denoted in black bars. Circle dots indicate the potential DNA binding sites, and a triangle indicates the predicted transcription start site of porcine BMP15; (B) PCR amplified fragments of truncated $B M P 15$ promoter (a) and the constructs digested by $\mathrm{Xho} I$ and Hind $I I I$ (b). M, 2000 bp DNA marker; and (C) Luciferase assays of the truncated constructs in $\mathrm{CHO}$, $\mathrm{C} 2 \mathrm{C} 12$, and NIH3T3 cells. The pGL3-Basic vector was used as control. Values are represented as mean $\pm \mathrm{SD}, * p<0.05, * * p<0.01, * * * p<0.001, n=3$.

The alignment of the DNA fragment between -357 to $-460 \mathrm{bp}$ region showed that this sequence was highly conserved among animal species and contained several potential DNA binding sites, including TFAP2C, LHX8/PITX1/NOBOX, and FOXO1 (Figure 4A). The consensus sequences of LHX8, PITX1, NOBOX, and FOXO1 were predicted by JASPAR program (jaspar.genereg.net) (Figure 4B). Two additional truncated constructs $\mathrm{pL0.4A}$ and $\mathrm{pL} 0.4 \mathrm{~B}$ were generated based on $\mathrm{pL} 0.4$ vector, in which the predicted TFAP2C site and LHX8/PITX1/NOBOX site was deleted, respectively (Figure 4Ca). Luciferase assays showed that TFAP2C site in pL0.4 and FOXO1 site in pL0.4B are not essential for maintaining BMP15 promoter activity, but LHX8/PITX1/NOBOX site in pL0.4A is a primary site that significantly affected the activity, because the deletion of LHX8/PITX1/NOBOX site can abolish BMP15 activity (Figure 4Cb). 

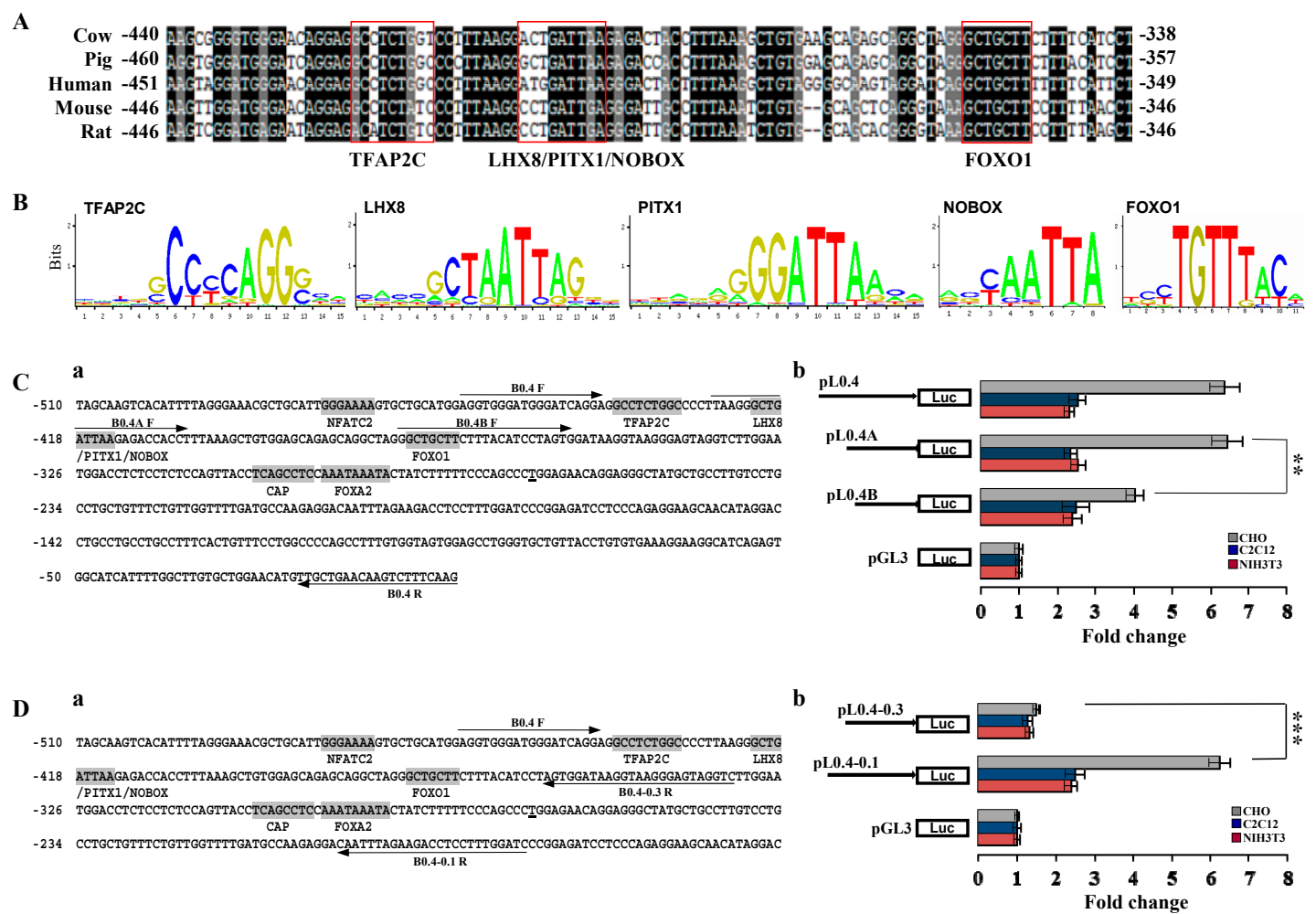

Figure 4. Functional analysis of regulatory elements in porcine BMP15 promoter. (A) Alignment of BMP15 promoter sequences among animal species. The potential DNA binding sites are highlighted in boxes; (B) The consensus sequences of TFAP2C, LHX8, PITX1, NOBOX, and FOXO1 are predicted by JASPAR program (jaspar.genereg.net); (C) The proximal sequence $(-460$ to $-1 \mathrm{bp})$ of $B M P 15$ promoter has multiple potential DNA binding sites (a). Luciferase assays were performed with different deletion constructs in $\mathrm{CHO}, \mathrm{C} 2 \mathrm{C} 12$, and NIH3T3 cells (b); and (D) Partial proximal sequence ( -460 to $-175 \mathrm{bp}$ ) of BMP15 promoter region (a). Luciferase assays were performed with different deletion constructs in $\mathrm{CHO}, \mathrm{C} 2 \mathrm{C} 12$, and $\mathrm{NIH} 3 \mathrm{~T} 3$ cells (b). The primer sequences used to generate the deletion are indicated by arrows. Values are represented as mean $\pm \mathrm{SD}$; $* * p<0.01 ; * * * p<0.001 ; n=3$.

To investigate if the proximal promoter sequence could influences BMP15 activity, two relevant reporter vectors, pL0.4-0.1 that retains the partial 5' UTR sequence and CAP and FOXA2 sites ( -460 to $-175 \mathrm{bp}$ ) and pL0.4-0.3 that has no CAP and FOXA2 sites ( -460 to $-332 \mathrm{bp}$ ), were constructed (Figure 4Da). Luciferase assays showed that partially removing $5^{\prime}$ UTR sequence in pL0.4-0.1 did not affect BMP15 promoter activity; however, the deletion of CAP and FOXA2 sites pL0.4-0.3 significantly eliminated BMP15 promoter activity (Figure 4Db). These results indicate that the transcription initiation site exists in between -332 to $-175 \mathrm{bp}$ and this area is essential for BMP15 activation.

\subsection{LIM Homeobox 8 (LHX8) Regulates BMP15 Promoter Activation}

The previous reports have showed that LHX8, NOBOX, and PITX1 are critical factors for the maintenance of oocytes and folliculogenesis [18,24,25]. To investigate whether these genes were 
expressed in porcine ovary, we prepared mRNA samples from nine porcine tissues and performed the RT-PCR. Results showed that both $L H X 8$ and BMP15 genes highly expressed in ovary tissue, but were undetectable in eight other tissues; PITX1 was expressed in ovary and lung; and NOBOX in ovary and testis. The expression of FOXO1, a gene that plays a wide range of roles in metabolism, cell cycle and tissue differentiation, for instance myogenic growth and differentiation [26,27], was detected in all somatic tissues (Figure 5A). Thus, we cloned and constructed the LHX8 expression vector pEC1-LHX8. The GFP-LHX8 fusion protein was observed in cellular nuclei, indicating that GFP-LHX8 fusion protein retains the translocation ability (Figure 5B). To determine the regulatory function of LHX8, the vectors pEC1-LHX8 and pL0.4A were cotransfected into CHO and NIH3T3 cells. The activity of the BMP15 promoter was significantly enhanced by overexpression of LHX8 (Figure 5C). This result demonstrates that LHX8 is a potential positive regulator in the upstream signaling pathway of porcine BMP15 gene. Because LHX8 was highly expressed in porcine ovary tissue (Figure 5A) and required for oogenesis in mouse [28], we speculate that LHX8 may promote $B M P 15$ gene expression in porcine ovary.

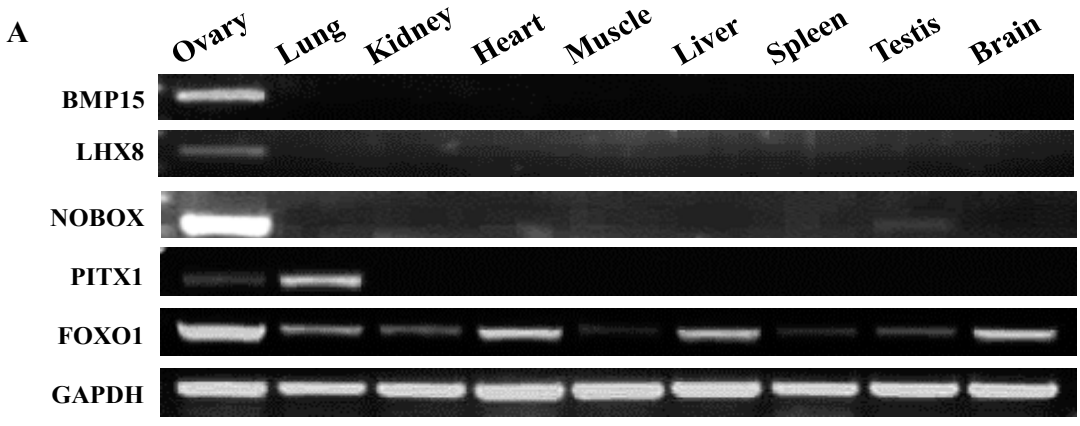

B

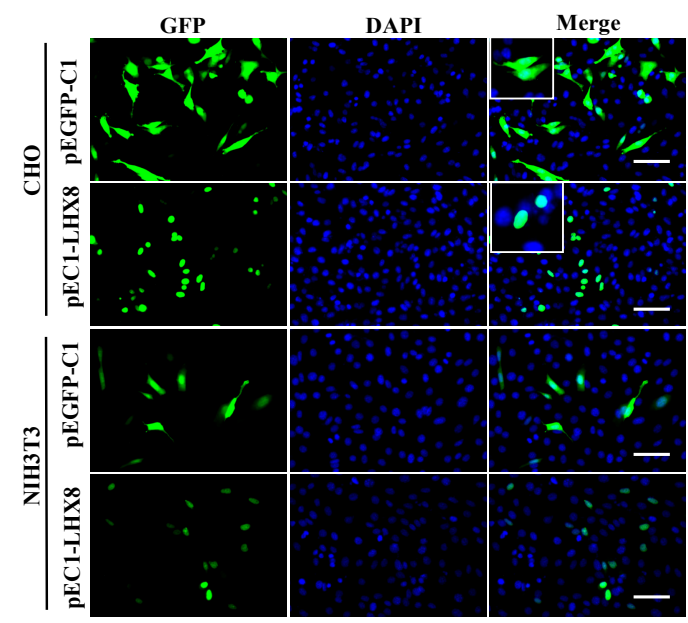

C
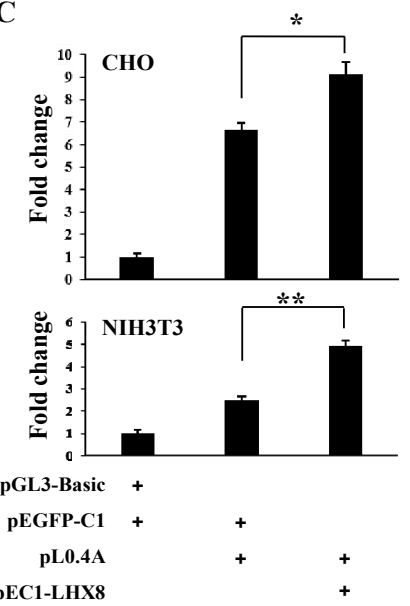

Figure 5. LHX8 enhances the activation of BMP15 promoter. (A) RT-PCR analysis of expression of transcription factors in different porcine tissues; (B) Overexpression of LHX8 in CHO and NIH3T3 cells. The plasmid pEGFP-C1 was used as control. Scale bar, $100 \mu \mathrm{m}$; and (C) Luciferase assays of BMP15 promoter activity that was enhanced by LHX8 were performed by cotransfection of vectors into CHO and NIH3T3 cells for $36 \mathrm{~h}$. + , indicats a vector was transfected into the recipient cells. Values are represented as mean \pm SD. $* p<0.05 ; * * p<0.01 ; n=3$. 


\section{Discussion}

In sheep and human the mutation and deletion of BMP15 gene could affect the response to controlled ovarian hyperstimulation and the birth weight, growth rate and carcass quality of lambs [29-31]. Thus, this gene is crucial for the ovarian function. The regulatory function and signaling pathway of BMP15 were not well illustrated in pig. Therefore, in this study, we investigated the promoter sequence of porcine BMP15 and demonstrated the potential regulatory elements that are able to be bound by transcription factors and enhance the BMP15 expression.

The previous studies of in situ hybridization and immunocytochemistry confirmed the exclusive oocyte localization of mouse Bmp15 in the time of the preovulatory LH surge. Relative oocyte expression levels of Bmp15 decreased significantly after PMSG + hCG treatment [32]. In human, the expression level of BMP15 mRNAs, which was highly expressed in high-quality embryos, was closely associated with oocyte maturation, fertilization, embryo quality, and pregnancy outcome [33]. Our previous study of BMP15 expression in different porcine tissues (ovary, lung, kidney, heart, muscle, liver, spleen, testis, and brain) also showed that BMP15 was only expressed in ovary [12]. To investigate the expression regulation of porcine $B M P 15$ gene, we constructed the porcine $B M P 15$ reporter vectors $\mathrm{pE} 2.2$ and $\mathrm{pL} 2.2$. Since a porcine ovarian cell line is not available and the primary-cultured ovarian cells are difficult to be obtained and handled, we, alternatively, used a CHO cell line to perform BMP15 assays using pE2.2 and pL2.2. The CHO cell line was derived from the ovary of the Chinese hamster, though it is an immortalized cell line that may not physiologically represent the ovarian epithelia, $\mathrm{CHO}$ cells were used to study Bmp15 promoter activation and signaling pathways [17,34]. The results of promoter activation confirmed that the porcine BMP15 promoter was exclusively activated in $\mathrm{CHO}$ cells, but not in myoblasts and fibroblasts, indicating that the cloned $B M P 15$ promoter retains the cell-type specificity.

Based on bioinformatics analysis and promoter deletion analysis of this study, we found that within the -427 to -376 bp region of the pig BMP15 promoter there exists sites for transcription factors LHX8, NOBOX, and PITX1, which are the potential positive regulators to activate pig BMP15. Although $L H X 8, N O B O X, B M P 15$, and GDF9 are highly expressed during ovarian follicle development [25,35], the definite regulatory network among these genes is under investigation. Several studies have reported an association between the BMP15 and premature ovarian failure, showing that the variant $B M P 15$ c. $-9 C>G$ results in modification of its binding sites for PITX1 that trans-activates BMP15 promoter and increases the recruitment of an elevated number of follicles/oocytes early in a woman's life $[18,30,36]$. Recent report showed that NOBOX was capable of up-regulating the GDF9 promoter during human fetal ovary development [37]. Nobox is a downstream target gene of SOHLH1 because in Sohlh1 null oocytes Nobox expression is defective resulting in a loss of genes downstream of the NOBOX pathway, such as Gdf9 and Oct4 [28]. Lhx8 is also a direct transcriptional target of SOHLH1. In $L h x 8^{-/-}$mouse, ovaries fail to maintain the primordial follicles, and the transition from primordial to growing follicles does not occur, in which the expression of oocyte-specific genes, such as $G d f 9$, Bmp 15 and $O c t 4$, are significantly reduced [25]. In $L h x 8$ conditional knockout (cKO) oocytes, Lhx 8 represses Lin28a expression indirectly interacting with the PI3K-AKT pathway and blocking the primary to secondary follicle transition [38]. On the other hand, in $Y y 1$, a multifaceted polycomb group gene, cKO oocytes exhibit reduced expressions of $L h x 8$, Gdf9 and Bmp15, indicating that Yyl function 
is required at each of these genes to act as an activator [20]. However, the genes downstream of LHX8 in the oocyte are still unclear [28]. We in this study demonstrated that LHX8 could significantly promote the activity of pig BMP15, indicating that LHX8 is an upstream positive factor of BMP15. Further investigation is needed to verify whether LHX8 can directly bind to BMP15 promoter and play a role in the regulation of folliculogenesis and granulosa cell growth in pig.

In addition, we found that the porcine BMP15 promoter did not have the typical TATA box sequence. The results of promoter deletion analysis indicated that the sequence within -332 to $-175 \mathrm{bp}$ region had a CAP site (-303) and a transcription start site for pig BMP15 gene, which was consistent with the transcription start site of rat BMP15 [39,40].

In conclusion, a key consensus sequence is identified within -427 to $-376 \mathrm{bp}$ region in porcine BMP15 promoter. The transcription factor LHX8 can specifically enhance BMP15 promoter activation. Understanding of LHX8 to BMP15 transactivation signaling can facilitate in vitro maturation of porcine oocytes, which are widely used for porcine cloning, because BMP15 treatment in vitro could promote porcine oocyte maturation and cumulus expansion [5]. In the future, the electrophoretic mobility shift assay (EMSA) and mRNA knock-down assay may be applied to directly prove the LHX8 regulatory function.

\section{Experimental Methods}

\subsection{Cell Culture}

Chinese hamster ovary (CHO) cells were grown in DMEM/F-12 medium (Gibco, Grand Island, NY, USA) with 10\% fetal bovine serum (FBS, HyClone, Logan, UT, USA). NIH3T3 fibroblasts and $\mathrm{C} 2 \mathrm{C} 12$ myoblasts were cultured in DMEM (Gibco) with 10\% FBS. All these cells were growing at $37{ }^{\circ} \mathrm{C}, 5 \% \mathrm{CO}_{2}$ in a humidified atmosphere and the media were changed every 2 days.

\subsection{Gene Cloning and Vector Construction}

To construct luciferase reporter vector, a 2166 bp (ENSSSCG00000012310) DNA fragment from pE2.2 vector that was constructed by this laboratory as described previously [12] was subcloned into pGL3-basic (Promega, Madison, WI, USA) at sites of XhoI and Hind $I I I$ and formed the reporter vector pL2.2. A series of truncated DNA fragments from $2.2 \mathrm{~kb}$ sequence were listed in Table S1 and used to construct reporter vectors that were then used to screen primary regulatory sequences of porcine BMP15 promoter.

The coding DNA sequence (CDS) of LIM homeobox 8 gene ( $L H X 8)$ was cloned from porcine ovary, inserted into the pGEM-T Easy Vector (Promega), and confirmed by DNA sequencing. LHX8 $\mathrm{CDS}$ was then subcloned into $\mathrm{Xho} / \mathrm{BamH} I$ sites of pEGFP-C1 (Invitrogen, Camarillo, CA, USA) to generate pEC1-LHX8 that could express GFP-LHX8 fusion protein.

\subsection{Reverse Transcription-Polymerase Chain Reaction (RT-PCR)}

Total RNAs were extracted from porcine ovaries by TRIzol (Invitrogen) according to the manufacturer's instructions. The total RNAs were examined by detecting the OD260/280 ratio and the RNAs with a ratio of 2.0 were then used for reverse transcription. One microgram RNAs were reverse 
transcribed using oligo-dT primers, reverse inhibitor, reverse transcriptase and RNA-free water (Thermo Scientific, Waltham, MA, USA). PCR reactions using PhantaTM Super-Fidelity DNA Polymerase kit (Vazyme, Nanjing, China) in a $50 \mu \mathrm{L}$ reaction volume were performed for 35 cycles at $94{ }^{\circ} \mathrm{C} 30 \mathrm{~s}, 60{ }^{\circ} \mathrm{C} 30 \mathrm{~s}$, and $72{ }^{\circ} \mathrm{C} 40 \mathrm{~s}$. The products of RCR were separated by $1.5 \%$ agarose gel electrophoresis. GAPDH was used as internal reference. Primers used in this study were listed in Table S2.

\subsection{Biological Assays}

To verify the tissue specificity of porcine $B M P 15$ promoter, $2 \times 10^{4}$ cells were seeded in a 48 -well plate $24 \mathrm{~h}$ before transfection. When these cells reached $80 \%$ confluence, the plasmids of pL2.2 and the control pRL-TK (10 ng) were cotransfected into CHO, NIH3T3, and C2C12 cells, respectively, by Lipofectamine 2000 Reagent (Invitrogen). For GFP fluorescence assay, the pE2.2 vector was transfected into cells for $48 \mathrm{~h}$, and then the cells were fixed by $4 \%$ paraformaldehyde in PBS for 10 min. After washing 3 times with PBS, nuclei were stained with DAPI (Sigma-Aldrich, St. Louis, MO, USA), and the fluorescence images were documented with the fluorescence microscope (Nikon, Tokyo, Japan). To screen the primary regulatory sequence of BMP15 promoter, the truncated constructs, including pL1.8, pL1.3, pL0.8, pL0.5, pL0.4, pL0.3 and pL0.2, were transfected into CHO, $\mathrm{C} 2 \mathrm{C} 12$ and NIH3T3 cells, respectively. In order to determine whether the GFP-LHX8 fusion protein located in cellular nuclei, pEC1-LHX8 and pEGFP-C1 was transfected into CHO and 3T3 cells for $48 \mathrm{~h}$, and then the cells were fixed by 4\% paraformaldehyde in PBS for $10 \mathrm{~min}$. After washing 3 times with PBS, nuclei were stained with DAPI and the fluorescence images were documented with the fluorescence microscope. To verify the interaction of LHX8 with the BMP15 promoter, pL0.4A construct was cotransfected with pEC1-LHX8 into CHO and $3 \mathrm{~T} 3$ cells, respectively.

For dual-luciferase assays, cells were harvested at $36 \mathrm{~h}$ after liposome-mediated transfection and lysed for $15 \mathrm{~min}$ at room temperature. Luciferase activity was stimulated by adding luciferase assay reagents (Promega) and then detected by a BHP9504 Luminometer (Hamamatsu, Japan). The average ratio of firefly luciferase light units to Renilla luciferase light units was calculated and we conducted three independent experiments and then collected the data for analysis. Statistical significance was accepted at $p<0.05$ and determined using one-way ANOVA followed by post-hoc tests (Newman-Keuls method).

\subsection{Bioinformatics Analysis}

The online programs of MatInspector function of Genomatix Suite (www.genomatix.de), JASPAR (www.jaspar.genereg.net), and Gene Regulation [41] were used to predict the potential primary regulatory region and transcription factor binding sites on BMP15 promoter ( -2166 to $-1 \mathrm{bp}$ ). ClustalW2 software (http://www.ebi.ac.uk) was used for multiple alignments of DNA sequences from different animal species (Cow, ENSBTAG00000045782; Pig, ENSSSCG00000012310; Human, ENSG00000130385; Mouse, ENSMUSG00000023279; Rat, ENSRNOG00000002984). 


\subsection{Statistical Analysis}

One-way ANOVA was used and Newman-Keuls multiple range tests were conducted as post-hoc tests if a significant difference $(p<0.05)$ existed in a group of data. Values were represented as the mean $\pm \mathrm{SD}$, and statistical significance was indicated as follows: ${ }^{*} p<0.05 ; * * p<0.01 ; * * * p<0.001$. Data were representative of at least three independent experiments.

\section{Supplementary Materials}

Supplementary materials can be found at http://www.mdpi.com/1422-0067/16/10/25759/s1.

\section{Acknowledgments}

We thank Fan Yang for the bioinformatics analysis and comments. This work was supported by the National Natural Science Foundation of China (31371505 and 31571521).

\section{Author Contributions}

Qianhui Wan and Huayan Wang conceived and designed the experiments; Qianhui Wan and Yaxian Wang performed the experiments and analyzed the data; Qianhui Wan and Huayan Wang wrote the paper.

\section{Conflicts of Interest}

The authors declare no conflict of interest.

\section{References}

1. Sun, X.J.; Mei, S.Q.; Tao, H.; Wang, G.D.; Su, L.N.; Jiang, S.W.; Deng, C.Y.; Xiong, Y.Z.; Li, F.G. Microarray profiling for differential gene expression in PMSG-hCG stimulated preovulatory ovarian follicles of Chinese Taihu and Large White sows. BMC Genom. 2011, 12, 111.

2. McNatty, K.P.; Moore, L.G.; Hudson, N.L.; Quirke, L.D.; Lawrence, S.B.; Reader, K.; Hanrahan, J.P.; Smith, P.; Groome, N.P.; Laitinen, M.; et al. The oocyte and its role in regulating ovulation rate: A new paradigm in reproductive biology. Reproduction 2004, 128, 379-386.

3. Emori, C.; Sugiura, K. Role of oocyte-derived paracrine factors in follicular development. Anim. Sci. J. 2014, 85, 627-633.

4. Knight, P.G.; Glister, C. TGF- $\beta$ superfamily members and ovarian follicle development. Reproduction 2006, 132, 191-206.

5. Lin, Z.L.; Li, Y.H.; Xu, Y.N.; Wang, Q.L.; Namgoong, S.; Cui, X.S.; Kim, N.H. Effects of growth differentiation factor 9 and bone morphogenetic protein 15 on the in vitro maturation of porcine oocytes. Reprod. Domest. Anim. 2014, 49, 219-227.

6. Dragovic, R.A.; Ritter, L.J.; Schulz, S.J.; Amato, F.; Thompson, J.G.; Armstrong, D.T.; Gilchrist, R.B. Oocyte-secreted factor activation of SMAD 2/3 signaling enables initiation of mouse cumulus cell expansion. Biol. Reprod. 2007, 76, 848-857. 
7. Peng, J.; Li, Q.; Wigglesworth, K.; Rangarajan, A.; Kattamuri, C.; Peterson, R.T.; Eppig, J.J.; Thompson, T.B.; Matzuk, M.M. Growth differentiation factor 9:bone morphogenetic protein 15 heterodimers are potent regulators of ovarian functions. Proc. Natl. Acad. Sci. USA 2013, 110, E776-E785.

8. Wu, X.; Matzuk, M.M. GDF-9 and BMP-15: Oocyte organizers. Rev. Endocr. Metab. Disord. 2002, 3, 27-32.

9. Galloway, S.M.; McNatty, K.P.; Cambridge, L.M.; Laitinen, M.P.E.; Juengel, J.L.; Jokiranta, T.S.; McLaren, R.J.; Luiro, K.; Dodds, K.G.; Montgomery, G.W.; et al. Mutations in an oocyte-derived growth factor gene (BMP15) cause increased ovulation rate and infertility in a dosage-sensitive manner. Nat. Genet. 2000, 25, 279-283.

10. Demars, J.; Fabre, S.; Sarry, J.; Rossetti, R.; Gilbert, H.; Persani, L.; Tosser-Klopp, G.; Mulsant, P.; Nowak, Z.; Drobik, W.; et al. Genome-wide association studies identify two novel BMP15 mutations responsible for an atypical hyperprolificacy phenotype in sheep. PLoS Genet. 2013, 9, e1003482.

11. Crawford, J.L.; McNatty, K.P. The ratio of growth differentiation factor 9: Bone morphogenetic protein 15 mRNA expression is tightly co-regulated and differs between species over a wide range of ovulation rates. Mol. Cell. Endocrinol. 2012, 348, 339-343.

12. Qin, M.; Wei, J.; Yu, X.; Zhang, J.; Liu, X.; Ma, X.; Wang, H. Construction and specificity of porcine Bmpl5 gene reporter vector. Sheng Wu Gong Cheng Xue Bao 2014, 30, 203-212.

13. Persani, L.; Rossetti, R.; di Pasquale, E.; Cacciatore, C.; Fabre, S. The fundamental role of bone morphogenetic protein 15 in ovarian function and its involvement in female fertility disorders. Hum. Reprod. Update 2014, 20, 869-883.

14. Margulis, S.; Abir, R.; Felz, C.; Nitke, S.; Krissi, H.; Fisch, B. Bone morphogenetic protein 15 expression in human ovaries from fetuses, girls, and women. Fertil. Steril. 2009, 92, 1666-1673.

15. Li, H.K.; Kuo, T.Y.; Yang, H.S.; Chen, L.R.; Li, S.S.; Huang, H.W. Differential gene expression of bone morphogenetic protein 15 and growth differentiation factor 9 during in vitro maturation of porcine oocytes and early embryos. Anim. Reprod. Sci. 2008, 103, 312-322.

16. Otsuka, F.; McTavish, K.J.; Shimasaki, S. Integral role of GDF-9 and BMP-15 in ovarian function. Mol. Reprod. Dev. 2011, 78, 9-21.

17. Lan, Z.J.; Gu, P.; Xu, X.; Jackson, K.J.; DeMayo, F.J.; O’Malley, B.W.; Cooney, A.J. GCNF-dependent repression of BMP-15 and GDF-9 mediates gamete regulation of female fertility. Embo J. 2003, 22, 4070-4081.

18. Fonseca, D.J.; Ortega-Recalde, O.; Esteban-Perez, C.; Moreno-Ortiz, H.; Patino, L.C.; Bermudez, O.M.; Ortiz, A.M.; Restrepo, C.M.; Lucena, E.; Laissue, P. BMP15 c.-9C >G promoter sequence variant may contribute to the cause of non-syndromic premature ovarian failure. Reprod. Biomed. Online 2014, 29, 627-633.

19. Jagarlamudi, K.; Rajkovic, A. Oogenesis: Transcriptional regulators and mouse models. Mol. Cell. Endocrinol. 2012, 356, 31-39.

20. Griffith, G.J.; Trask, M.C.; Hiller, J.; Walentuk, M.; Pawlak, J.B.; Tremblay, K.D.; Mager, J. Yin-yang1 is required in the mammalian oocyte for follicle expansion. Biol. Reprod. 2011, 84, 654-663. 
21. Goodrich, J.A.; Tjian, R. Unexpected roles for core promoter recognition factors in cell-type-specific transcription and gene regulation. Nat. Rev. Genet. 2010, 11, 549-558.

22. Zhao, X.; Mo, D.L.; Li, A.N.; Gong, W.; Zhang, Y.; Qian, W.W.; Chen, W.Q.; Xiao, S.Q.; Chen, Y.S. Characterization and transcriptional regulation analysis of the porcine PAQR6 gene. DNA Cell Biol. 2011, 30, 947-954.

23. Tao, H.; Mei, S.; Zhang, X.; Peng, X.; Yang, J.; Zhu, L.; Zhou, J.; Wu, H.; Wang, L.; Hua, L.; et al. Transcription factor $\mathrm{C} / \mathrm{EBP} \beta$ and $17 \beta$-estradiol promote transcription of the porcine p53 gene. Int. J. Biochem. Cell Biol. 2014, 47, 76-82.

24. Tarnawa, E.D.; Baker, M.D.; Aloisio, G.M.; Carr, B.R.; Castrillon, D.H. Gonadal expression of FOXO1, but not FOXO3, is conserved in diverse Mammalian species. Biol. Reprod. 2013, 88, 103.

25. Choi, Y.; Ballow, D.J.; Xin, Y.; Rajkovic, A. Lim homeobox gene, LHX8, is essential for mouse oocyte differentiation and survival. Biol. Reprod. 2008, 79, 442-449.

26. Puthanveetil, P.; Wan, A.; Rodrigues, B. FOXO1 is crucial for sustaining cardiomyocyte metabolism and cell survival. Cardiovasc. Res. 2013, 97, 393-403.

27. Pandey, A.; Kumar, G.S.; Kadakol, A.; Malek, V.; Gaikwad, A.B. FOXO1 inhibitors: The future medicine for metabolic disorders? Curr. Diabetes Rev. 2015, in press.

28. Pangas, S.A.; Choi, Y.; Ballow, D.J.; Zhao, Y.G.; Westphal, H.; Matzuk, M.M.; Rajkovic, A. Oogenesis requires germ cell-specific transcriptional regulators Sohlh1 and LHX8. Proc. Natl. Acad. Sci. USA 2006, 103, 8090-8095.

29. Abir, R.; Fisch, B. Invited commentary: A single nucleotide polymorphism in BMP15 is associated with high response to controlled ovarian hyperstimulation. Reprod. Biomed. Online 2011, 23, 77-80.

30. Hanevik, H.I.; Hilmarsen, H.T.; Skjelbred, C.F.; Tanbo, T.; Kahn, J.A. A single nucleotide polymorphism in BMP15 is associated with high response to ovarian stimulation. Reprod. Biomed. Online 2011, 23, 97-104.

31. Roche, A.; Ripoll, G.; Joy, M.; Folch, J.; Panea, B.; Calvo, J.H.; Alabart, J.L. Effects of the Fec $X^{R}$ allele of BMP15 gene on the birth weight, growth rate and carcass quality of Rasa Aragonesa light lambs. Small Rumin. Res. 2012, 108, 45-53.

32. Mester, B.; Ritter, L.J.; Pitman, J.L.; Bibby, A.H.; Gilchrist, R.B.; McNatty, K.P.; Juengel, J.L.; McIntosh, C.J. Oocyte expression, secretion and somatic cell interaction of mouse bone morphogenetic protein 15 during the peri-ovulatory period. Reprod. Fertil. Dev. 2015, 27, 801-811.

33. Li, Y.; Li, R.Q.; Ou, S.B.; Zhang, N.F.; Ren, L.; Wei, L.N.; Zhang, Q.X.; Yang, D.Z. Increased GDF9 and BMP15 mRNA levels in cumulus granulosa cells correlate with oocyte maturation, fertilization, and embryo quality in humans. Reprod. Biol. Endocrin 2014, 12, 81.

34. Moore, R.K.; Otsuka, F.; Shimasaki, S. Molecular basis of bone morphogenetic protein-15 signaling in granulosa cells. J. Biol. Chem. 2003, 278, 304-310.

35. Park, M.; Jeon, S.; Jeong, J.H.; Park, M.; Lee, D.R.; Yoon, T.K.; Choi, D.H.; Choi, Y. Identification and Characterization of LHX8 DNA Binding Elements. Balsaenggwa Saengsig 2012, 16, 379-384.

36. Abir, R.; Fisch, B.; Johnson, M.H. BMP15, fertility and the ovary. Reprod. Biomed. Online 2014, $29,525-526$. 
37. Bayne, R.A.L.; Kinnell, H.L.; Coutts, S.M.; He, J.; Childs, A.J.; Anderson, R.A. GDF9 is transiently expressed in oocytes before follicle formation in the human fetal ovary and is regulated by a novel NOBOX transcript. PLoS ONE 2015, 10, e0119819.

38. Ren, Y.; Suzuki, H.; Jagarlamudi, K.; Golnoski, K.; McGuire, M.; Lopes, R.; Pachnis, V.; Rajkovic, A. LHX8 regulates primordial follicle activation and postnatal folliculogenesis. BMC Biol. 2015, 13, 39.

39. Edwards, S.J.; Reader, K.L.; Lun, S.; Western, A.; Lawrence, S.; McNatty, K.P.; Juengel, J.L. The cooperative effect of growth and differentiation factor-9 and bone morphogenetic protein (BMP)-15 on granulosa cell function is modulated primarily through BMP receptor II. Endocrinology 2008, 149, 1026-1030.

40. Jaatinen, R.; Laitinen, M.P.; Vuojolainen, K.; Aaltonen, J.; Louhio, H.; Heikinheimo, K.; Lehtonen, E.; Ritvos, O. Localization of growth differentiation factor-9 (GDF-9) mRNA and protein in rat ovaries and cDNA cloning of rat GDF-9 and its novel homolog GDF-9B. Mol. Cell. Endocrinol. 1999, 156, 189-193.

41. Gene Regulation-Programs. Available online: www.gene-regulation.com/pub/programs.html (accessed on 2 July 2015).

(C) 2015 by the authors; licensee MDPI, Basel, Switzerland. This article is an open access article distributed under the terms and conditions of the Creative Commons Attribution license (http://creativecommons.org/licenses/by/4.0/). 